\title{
¿Crisis financiera o de gestión? Evolución del sector salud desde la perspectiva de los entes territoriales
}

\author{
Is it a financial or a management crisis? \\ Evolution of the health sector from the \\ perspective of territorial entities
}

Crise financeira ou da gestão? Evolução do sector da saúde a partir da perspectiva dos territórios

\author{
Andrés Camilo Santos Ospina* \\ Mario García Molina** \\ Liliana Chicaiza Becerra ${ }^{* * *}$
}

Fecha de recepción: 18 de agosto de 2015

Concepto de evaluación: 6 de noviembre de 2015

Fecha de aprobación: 9 de diciembre de 2015

\footnotetext{
Magíster en Ciencias Económicas. Docente ocasional. Facultad de Ciencias Económicas, Universidad Nacional de Colombia. Bogotá, Colombia. ; Dirección postal: Carrera 45 con Avenida NQS, ciudad universitaria, edificio 311, Bogotá, Colombia. Correo electrónico: acsantosos@unal.edu.co

* PhD en Economía. Profesor titular. Facultad de Ciencias Económicas. Universidad Nacional de Colombia. Profesor-investigador, Universidad Externado de Colombia. Bogotá, Colombia. Correo electrónico: mgarciamo@ unal.edu.co

*** PhD en Economía y Gestión de la Salud. Profesora titular. Facultad de Ciencias Económicas. Universidad Nacional de Colombia. Bogotá, Colombia. Correo electrónico: lachicaizab@unal.edu.co
} 


\title{
Resumen
}

Tradicionalmente, los problemas asociados al sector salud en los entes territoriales se han enmarcado principalmente en un plano financiero. En esa línea los problemas de eficiencia se han venido tratando con medidas fiscales o financieras. El artículo presenta la situación financiera del sector salud desde los entes territoriales, para ello estudia la normatividad y la evolución de las principales cifras entre 2010 y 2014. El superávit de los últimos años de los fondos departamentales de salud sugiere que la política pública debería trascender de lo financiero y concentrarse en el fortalecimiento de herramientas institucionales y de gestión que permitan mejorar los resultados en salud.

Palabras clave: salud, entidades territoriales, crisis, gestión.

Clasificación JEL: H41 H51 H75 I13 I18.

\begin{abstract}
The problems of the health sector in the territorial entities have mainly been seen from a financial perspective. Thus, efficiency problems have been treated with fiscal and financial measures. This article presents the financial situation of the health sector from the territorial entities perspective, which includes the study of regulation and the main financial facts between 2010 and 2014. In the last years local health funds have had a financial surplus, which suggests that public policy should transcend the financial issue and concentrate on strengthening institutional and management tools to improve health results.
\end{abstract}

Keywords: health, territorial entities, crisis, management. 


\section{Resumo}

Tradicionalmente os problemas associados ao setor da saúde nos territórios têm marcado principalmente um plano financeiro. Nessa linha os problemas de eficiência vêm tentando com medidas fiscais ou financeiras. $\mathrm{O}$ artigo apresenta a situação financeira do sector da saúde por parte das autoridades locais, para este estudo da regulação e evolução das principais figuras entre 2010 e 2014. O superávit nos últimos anos de fundos de saúde departamentais sugere que a política pública deveria transcender do financeiro e concentrar-se no fortalecimento de instrumentos institucionais e de gestão para melhorar os resultados de saúde.

Palavras-chave: saúde, territórios, crises, gestão. 


\section{INTRODUCCIÓN}

La reforma que se realizó al sector salud durante el año 2015, al igual que las demás reformas de los últimos años, busca enfrentar una problemática en la que el protagonismo lo llevan los aspectos financieros. La concentración en lo financiero resulta en parte de una mirada desde el punto de vista central, en la que las variables monetarias agregadas resaltan fácilmente. El presente trabajo parte del supuesto de que la mirada desde los entes territoriales permite comprender mejor la complejidad del flujo de recursos e identificar los problemas que trascienden a lo meramente financiero.

Para comprender el problema desde una perspectiva territorial conviene comenzar con la Constitución Política de Colombia, la cual estableció principalmente dos cambios políticos de gran envergadura histórica: el primero, instaurar al país como un Estado social de derecho y, el segundo, la descentralización regional. Es claro que la descentralización les ha permitido a las entidades subnacionales contar con mayor autonomía administrativa y fiscal para el manejo de los recursos (Urrutia \& Llano, 2012; Bonet, Pérez \& Ayala, 2014). No obstante, la conjugación de estos principios constitucionales ha enmarcado también dos responsabilidades a cargo de las entidades territoriales: en primer lugar, garantizar a la población el suministro de bienes y servicios meritorios, en las cuales se incluirían la salud, educación, el agua potable y el saneamiento básico; la segunda responsabilidad es ejercer su autonomía conforme a las normas de responsabilidad fiscal, cuyo objetivo macro es lograr la sostenibilidad fiscal de las finanzas públicas territoriales.

Es cuestionable enmarcar un derecho constitucional como la salud frente a un objetivo de sostenibilidad fiscal. Sin embargo, se parte de la premisa 
económica de que los recursos monetarios y físicos son escasos y, por tanto, es imperativo lograr los mejores resultados posibles en la dotación efectiva de este servicio meritorio (Stiglitz, 2000). En esta medida, las entidades territoriales en pleno ejercicio de su autonomía deben conseguir eficiencia en el manejo de los recursos destinados a este sector, condición que implica legalidad y transparencia en la contratación, además de calidad y control en la ejecución de la inversión social.

En un marco institucional descentralizado, la importancia de la eficiencia de las entidades territoriales va más allá de ser fundamento de la sostenibilidad fiscal, porque tales entidades administran recursos públicos con destinación social y, por ende, las implicaciones socioeconómicas de su uso se podrían materializar en mayores niveles de salud que, además de ser un objetivo en sí mismos al aumentar el bienestar directamente, también pueden aportar de manera indirecta al permitir lograr mayores tasas de productividad, crecimiento y desarrollo económico.

Por otra parte, luego de un poco más de dos décadas de descentralización territorial, es imperativo que el análisis de la situación del sector salud no se concentre en el debate sobre la escasez de recursos para financiar la prestación de los servicios; tal como han señalado autores como Melo y Ramos (2010), se necesita avanzar también en la evaluación de si los recursos transferidos se asignan eficientemente. También es importante establecer si hay calidad en la ejecución de los mismos, y un acceso adecuado, de tal forma que los servicios efectivamente lleguen a la población y, por ende, sean catalizadores de mayor rentabilidad social.

El análisis del sector salud desde el nivel subnacional de Gobierno es trascendental no solo por el papel que ha tenido el régimen subsidiado en la búsqueda de la cobertura universal, sino también si se considera que las administraciones territoriales tienen una alta incidencia en el diseño y la ejecución de las políticas públicas en salud, y que cuentan con las competencias legales para realizar seguimiento y control sobre la prestación de los servicios.

En ese orden de ideas, este artículo busca presentar una aproximación a los antecedentes y estado actual del sector salud desde la perspectiva de las entidades territoriales. La metodología que se adoptará consistirá de dos partes: en primer lugar la revisión normativa del sector, que arranca de la consideración que el sistema de salud en Colombia se caracteriza por su amplia y compleja regulación; en segundo lugar se analiza el estado del sector a partir de sus cifras y de la crónica del período 2010-2014.

Así, el documento se divide en cinco secciones: en la primera se presenta un resumen de los antecedentes normativos del sector salud en las entidades 
territoriales; en la segunda sección se realiza un esbozo sobre la situación del sector, luego de la declarada crisis financiera del 2010; en la tercera se exhiben las perspectivas del sector desde el nivel subnacional de Gobierno; en la cuarta se presenta el balance presupuestal de los fondos departamentales de salud para el año 2014; y en la quinta se presentan, a modo de conclusiones, las reflexiones finales.

\section{LA NORMATIVIDAD EN CON- TEXTO}

El Gobierno nacional a través de la Ley 100 de 1993 reguló el Sistema General de Seguridad Social en Salud (SGSSS), el cual se divide principalmente en dos tipos de regímenes, que en principio son excluyentes entre sí: contributivo y subsidiado ${ }^{1}$. El régimen contributivo está conformado por todos aquellos individuos con capacidad económica para afiliarse al sistema mediante el pago periódico de una cotización. Por su parte, el régimen subsidiado está compuesto por todas las personas que por su vulnerabilidad económica y social no se encuentran afiliadas al régimen contributivo y, por lo tanto, el Estado los vincula por medio de una cotización subsidiada. El nuevo régimen fue defendido teóricamente con la idea del pluralismo estructurado (Londoño \& Frenk, 1997). ${ }^{2}$

La diferencia sustancial entre estos dos regímenes se ubicó particularmente en la conformación del Plan Obligatorio de Salud (POS), pues el Estado definió planes de beneficios diferenciales, que derivaron importantes implicaciones en la financiación de los mismos $\mathrm{y}$, por ende, en la cobertura de atención médica y suministro de medicamentos.

En el nivel territorial, el artículo 174 de la mencionada ley señaló que el SGSSS integra las instituciones de dirección, promoción y de prestación de servicios de todos los entes territoriales. En ese sentido, mediante la Ley 60 de 1993, el Gobierno nacional le otorgó a los gobiernos subnacionales competencias en materia de salud, facultándolos para organizar la red pública de salud y con ello suscribir contratos con las entidades promotoras de salud (EPS) dentro del régimen subsidiado, para garantizar la atención de la

1 Es de precisar que existe un régimen de excepción, en el cual se encuentran las fuerzas militares, la policía, los servidores públicos de Ecopetrol y de las universidades públicas, y los docentes afiliados al Fondo de Prestaciones Sociales del Magisterio.

2 La reforma colombiana fue influyente en otros países de América latina, como por ejemplo México, Argentina y República Dominicana (Frenk et al., 2006; Almeida, 2002; Bertranou, 1999; Castellanos, 2008). El caso de México es ilustrativo. El problema de las disparidades en el gasto a nivel territorial también ha aparecido allí (Knaul et al., 2012) pero no se pueden hacer comparaciones de manera directa porque el marco institucional es distinto. México tiene un régimen federal, mientras que Colombia tiene un régimen centralista. De allí la importancia de contar con estudios que exploren la problemática con atención a la normatividad. 
denominada población pobre y vulnerable de su respectiva jurisdicción.

Con la promulgación de la Ley 100 se tenía como objetivo principal ampliar la cobertura de afiliación, de tal manera que se alcanzara gradualmente la cobertura universal en salud. Política que paralelamente se debía sustentar con una estrategia de crecimiento económico y de formalización laboral que permitiera aumentar la afiliación al régimen contributivo y, con ello, la disminución progresiva de la financiación pública al régimen subsidiado, de forma que en el mediano plazo el sistema de salud fuera financieramente autosostenible.

No obstante lo anterior, finalizando la década de los 90 la situación fiscal territorial se hizo financieramente insostenible, dado el exorbitante crecimiento de los gastos, especialmente los correspondientes al rubro de funcionamiento, en proporción de los recursos propios generados por la entidad territorial. Adicionalmente, se dio un incremento sustancial de la deuda subnacional, del pasivo pensional y el aumento de la dependencia fiscal en relación con las transferencias provenientes del situado fiscal para la financiación de la inversión social (Ministerio de Hacienda y Crédito Público, 2009; Urrutia \& Llano, 2012; Dirección General de Apoyo Fiscal, 2014).

En lo concerniente al sector salud, el marco legal no resultaba claro respecto a la asignación de competencias para los diferentes niveles de Gobierno. Esta situación permitió a las administraciones territoriales ejecutar los recursos sin ningún criterio técnico de racionalidad y eficiencia, generando con ello la acumulación de déficit sectorial en el nivel central, que se profundizó con la creación deliberada de entidades descentralizadas subnacionales (hospitales públicos conformados como empresas sociales del estado y establecimientos públicos), cuyo marco regulatorio permitía a las entidades territoriales configurar una estructura administrativa sin viabilidad financiera y sin una sólida defensa jurídica, dado el incremento de las obligaciones contingentes derivadas de la acumulación de procesos judiciales en contra de los entes subnacionales que finalmente restringían la reorientación de recursos en favor de la inversión social en salud (Ministerio de Hacienda y Crédito Público, 2009; Nupia \& Sánchez, 2001).

En respuesta a esta crisis fiscal, el Gobierno central estableció un conjunto de normas de responsabilidad fiscal con el objetivo de: I) ajustar el endeudamiento público conforme a la capacidad financiera de las entidades territoriales -Ley 358 de 1997-; II) financiar el pasivo pensional mediante la creación de un fondo común territorial -Ley 549 de 1999-; III) intervenir económicamente las entidades territoriales insolventes financieramente mediante la suscripción de acuerdos de 
reestructuración de pasivos -Ley 550 de 1999-; IV) racionalizar los gastos de funcionamiento mediante la creación de categorías presupuestales con limites diferenciales de gastos en proporción de los ingresos corrientes de libre destinación -Ley 617 de 1900-; y IV) formalizar normas de responsabilidad y de transparencia fiscal, en particular reguló la implementación del marco fiscal de mediano plazo para los entes territoriales -Ley 819 de 2003- (Dirección General de Apoyo Fiscal, 2014).

Frente a la provisión de servicios públicos de salud y educación, entre otros, el Gobierno redefinió las competencias tanto para la nación como para las entidades del orden territorial a través de la Ley 715 de $2001^{3}$. Concretamente, para el caso del sector salud, la norma concedió a los gobiernos departamentales atribuciones para orientar la política sectorial, y también para asesorar, coordinar y monitorear las acciones de los municipios que hacen parte de su jurisdicción.

Como señalan Bonet, Perez y Ayala (2014), para garantizar el desarrollo efectivo de dichas competencias, la
Ley creó el Sistema General de Participaciones, cuyo marco definió la asignación de recursos que la nación debía transferir a las entidades territoriales para la financiación de los sectores de salud, educación, deporte, cultura, agua potable y saneamiento básico, entre otros ${ }^{4}$. De esta forma, organizó una distribución sectorial en la que establecía una participación con destinación específica para el sector salud equivalente al $24,5 \%$, otra para educación correspondiente al 58,5\% y una última participación de $17 \%$ para aquellas cuestiones que fueran de propósito general. Para esta última, cabe advertir que la Ley 1176 de $2007^{5}$ definió la participación en agua potable y saneamiento básico en 5,4\% y el restante $11,6 \%$ para los demás sectores que componen la participación de propósito general.

En lo concerniente a la participación para salud y en coherencia con las competencias definidas por la Ley 715 de 2001, se contempló que los recursos transferidos al sector se destinaran única y exclusivamente para la financiación de los siguientes componentes: en primer lugar, los subsidios a la

3 "Por la cual se dictan normas orgánicas en materia de recursos y competencias de conformidad con los artículos 151, 288, 356 y 357 de la Constitución Política y se dictan otras disposiciones para organizar la prestación de los servicios de educación y salud, entre otros".

4 Cabe precisar que frente al régimen anterior de transferencias, es decir la Ley 60 de 1993, la Ley 715 de 2001 desligó gradualmente las transferencias territoriales del comportamiento de los ingresos corrientes de la nación, al considerar principalmente los problemas de riesgo moral que se presentaban en el nivel subnacional, puesto que mejoras en el esfuerzo fiscal del Gobierno nacional central se traducían, vía mayores transferencias, en incrementos en el gasto público territorial con escasos criterios de eficiencia y calidad en la ejecución. Situación que contribuyó, en la década de los noventa, a la profundización de resultados fiscales deficitarios tanto en las entidades territoriales como en el consolidado nacional.

5 "Por la cual se desarrollan los artículos 356 y 357 de la Constitución Política y se dictan otras disposiciones." 
demanda a través de la afiliación progresiva de la población pobre al régimen subsidiado hasta lograr la cobertura universal; en segundo lugar, consignó expresamente la financiación de la prestación del servicio de salud a la población pobre en lo no cubierto con subsidios a la demanda; y finalmente, la inversión en acciones de salud pública, conforme a la prioridades definidas en Plan Nacional de Salud Pública del Ministerio de Salud.

Es de precisar que la mencionada Ley estableció a priori competencias para los departamentos y distritos respecto a la administración, financiación y cofinanciación de los tres componentes en salud: salud pública, régimen subsidiado, prestación de servicios a la población pobre no afiliada. Sin embargo, para el ámbito municipal solo definió competencias para los dos primeros, por lo que la última se dejó a cargo de las administraciones departamentales, con excepción de aquellos municipios que se hubieran certificado en salud ${ }^{6} \mathrm{y}$ que, por tanto, fueran responsables de la atención del primer nivel de complejidad y de las actividades No POS.

No obstante, la Ley 715 de 2001 estipuló que los departamentos deberían mantener coordinación con los municipios certificados de su jurisdicción, en cuanto a la cobertura en salud de la población y a la articulación de las instituciones hospitalarias municipales con las redes departamentales.

En lo concerniente al régimen subsidiado, posteriormente el Gobierno nacional, mediante la Ley 1122 de 2007', hizo expresas las fuentes de financiación de este régimen para las entidades territoriales. Para tal efecto puntualizó sobre los recursos del Sistema General de Participaciones, salud, los provenientes de ETESA $^{8}$, las rentas cedidas destinadas a funcionamiento, las rentas de destinación específica resultantes del esfuerzo propio territorial, así como también los recursos procedentes del Fondo de Solidaridad y Garantía (FOSYGA) y de las cajas de compensación familiar.

En el año 2008, a través del Decreto 028, el Gobierno estableció una estrategia de monitoreo, seguimiento y control integral al gasto que ejecutan las entidades territoriales con los recursos del Sistema General de Participaciones. El marco de la estrategia definió un total de diecisiete eventos que podían poner en riesgo la prestación adecuada

\footnotetext{
6 La Ley 1176 de 2007 hizo expresa la posibilidad para que aquellos municipios que al término de la expedición de la Ley 715 de 2001 no contaran con la certificación en salud, que podían adquirirla en cumplimiento de la reglamentación para tal efecto.

7 "Por la cual se hacen algunas modificaciones en el Sistema General de Seguridad Social en Salud y se dictan otras disposiciones".

8 Actualmente Coljuegos.
} 
de los servicios de salud, educación y los demás sectores que componen la participación de propósito general. Con este fin, configuró dos tipos de intervención: la medida preventiva y la medida correctiva. La primera se refiere a la suscripción de un plan de desempeño que contempla una serie de obligaciones para la entidad territorial con el fin de eliminar los eventos de riesgo identificados; la segunda contempla medidas más estrictas tales como la suspensión de giros, el giro directo de recursos, la asunción temporal de competencias y la suspensión de procesos contractuales (Ministerio de Hacienda y Crédito Público, 2011).

Para la aplicación de esta estrategia en los municipios, la norma establece que los departamentos tendrán la función de intermediación entre estos y la nación, con el fin de que acompañen el desarrollo de las medidas adoptadas en los entes territoriales de su jurisdicción.

Bajo este marco y, especialmente, en concordancia con el objetivo de lograr la cobertura universal en salud, las transferencias provenientes del Sistema General de Participaciones destinadas a la financiación del sector salud, se han concentrado en el aseguramiento de la población al régimen subsidiado, con la consecuente reducción gradual de los recursos que financian la prestación del servicio a la población pobre en lo no cubierto con subsidios a la demanda (PPNA) y que también financian, aunque en menor proporción, la estabilización de los recursos destinados a las acciones de Salud Pública (ver Figura 1).

Durante el periodo 2002-2013 las transferencias han registrado un crecimiento de 7,6 \% promedio anual. Esta tendencia incremental se explica esencialmente por lo dispuesto en el artículo 357 de la Constitución Política de Colombia, el cual inicialmente determinó un crecimiento anual equivalente al promedio de la variación porcentual de los ingresos corrientes de la nación de los últimos cuatro años. Sin embargo, posteriormente el Acto Legislativo 04 de $2007^{9}$ puntualizó que del 2008 al 2016 se incrementarían en un porcentaje igual al de la tasa de inflación causada, más una tasa de crecimiento real definida expresamente en la norma.

En general, en los últimos años ha sido creciente la tendencia de crecimiento de los recursos transferidos para el aseguramiento en Colombia, en particular por la ampliación de la cobertura al régimen subsidiado en el nivel subnacional de Gobierno. En donde especialmente las administraciones municipales han tenido un papel determinante, puesto que han venido ejerciendo funciones de asegurador de la población vulnerable. La herramienta

"Por el cual se reforman los artículos 356 y 357 de la Constitución Política." 
que se ha venido empleando para la identificación de la población beneficiaria del régimen subsidiado ha sido el Sistema de Identificación de Potenciales Beneficiarios de Programas Sociales (SISBÉN), cuyo funcionamiento ha sido efectivo toda vez que los niveles de aseguramiento en salud son cercanos a la cobertura universal.

Sin embargo, en los últimos años se ha venido cuestionando el desempeño del SISBÉN como instrumento de focalización del régimen subsidiado, puesto
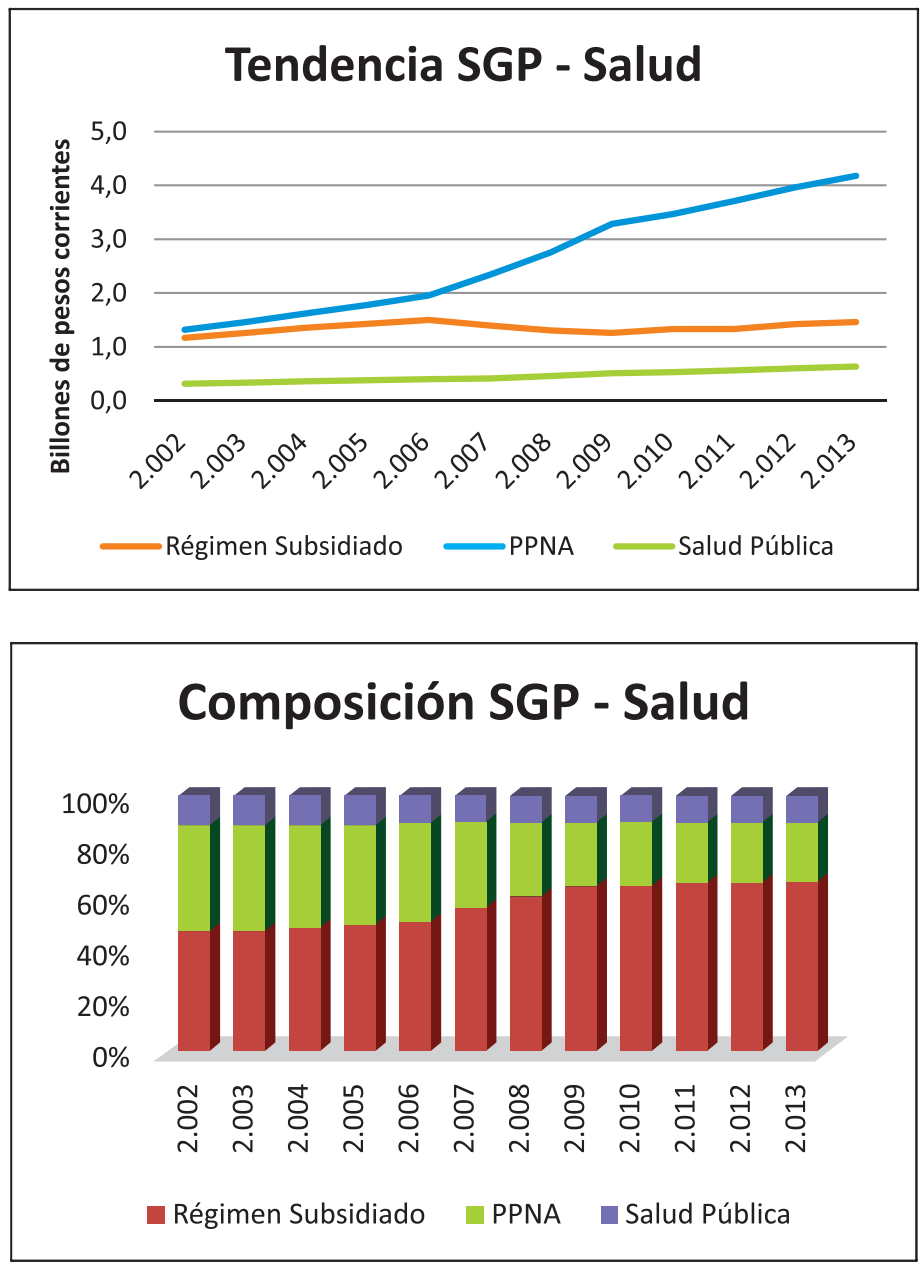

Figura 1. Composición y tendencia Sistema General de Participaciones SGP-Salud Fuente: elaboración propia con datos del Departamento Nacional de Planeación. 
que no ha estado ajeno a los procesos políticos y clientelistas del nivel territorial, promoviendo con ello el crecimiento de sesgos estratégicos en la información de las encuestas. En ese sentido, Bottia, Cardona-Sosa, y Medina (2012) presentan evidencia empírica sobre la presencia de hogares en Bogotá cuyas "respuestas estratégicas" les han permitido ser beneficiarios del régimen subsidiado, aun cuando sus condiciones económicas son relativamente mejores a las de la población realmente vulnerable. Como resultado, esta situación ha venido generando mayores costos para el sistema de salud y ha propiciado que el SISBÉN comience a ser visto como un instrumento regresivo.

CRISIS FINANCIERA DEL SECTOR SALUD: ¿QUÉ HA PASADO EN LAS ENTIDADES TERRITORIALES?

En el año 2010 explotó la crisis financiera del sector salud. La crisis se desató como producto de la creciente demanda por los servicios excluidos del plan obligatorio de salud (POS), que se profundizó cuando la Corte Constitucional, mediante Sentencia C-463 de 2008, obligó al FOSYGA a reconocer la totalidad de los recobros derivados de eventos No POS del régimen contributivo, siempre y cuando estos hubiesen sido aprobados por el denominado Comité Técnico Científico.
En el régimen subsidiado los recobros originados por estos eventos tuvieron que ser, en su mayor parte, asumidos por las administraciones departamentales, situación que afectó considerablemente las finanzas públicas territoriales, pues una porción considerable de estos pasivos no tenían respaldo presupuestal, por lo que se convirtieron en una fuente permanente de déficit fiscal. Obsérvese que, en principio, esta fuente de gasto no está bajo el control de los entes territoriales.

Para el año 2011, los departamentos registraron un déficit por prestación de servicios de aproximadamente $\$ 591.126$ millones. De esta cifra el 84 \% (\$496.345 millones) correspondía a facturación por prestación de servicios sin respaldo presupuestal y el restante $16 \%$ (\$94.781 millones) correspondía a recobros. Al incluir el déficit acumulado de vigencias anteriores, los departamentos presentaban un déficit presupuestal por prestación de servicios de $\$ 976$ mil millones (Dirección General de Apoyo Fiscal, 2015a).

Sumado a lo anterior, la sentencia de la Corte Constitucional T-760 de 2008 había ordenado la homologación de los planes de beneficios del régimen subsidiado y contributivo, cuyo modelo financiero obligó a las entidades territoriales a concentrar su capacidad institucional para controlar los problemas de selección adversa y riesgo moral, pues dada la unificación de los planes de aseguramiento existían 
incentivos para que contribuyentes del régimen contributivo pasarán al subsidiado y con ello obtener ahorros salariales bajo un nivel equivalente de atención en salud. Una situación como esta podía generar problemas en la financiación del régimen subsidiado, toda vez que al disminuir las contribuciones del régimen contributivo se reducirían simultáneamente los recursos provenientes de la subcuenta de solidaridad del FOSYGA (Núñez, Zapata, Castañeda, Fonseca \& Ramírez, 2012).

En este contexto, la crisis del sector hacía evidentes fallas estructurales, cuyas causas se atribuían principalmente a un problema de carácter financiero pero con notables repercusiones sobre los principios de eficiencia, calidad y equidad en la prestación de los servicios de salud. Una de las medidas adoptadas por el Gobierno nacional fue aumentar los recursos asignados al sector. Muestra de ello fue la declaración de emergencia social (que posteriormente se declaró inexequible), cuyo marco amplió los recursos para el sector, en particular provenientes de impuestos sobre los juegos de suerte y azar, así como el consumo de cigarrillo y licores, entre otros. Se trataba de rentas de destinación específica que posteriormente fueron reglamentadas y declaradas permanentes mediante la Ley 1393 de $2010^{10}$.

Con la promulgación de la Ley 1438 de $2011^{11}$, el Gobierno nacional ratificó las competencias de los entes territoriales respecto al seguimiento y control del aseguramiento de los afiliados al régimen subsidiado, pero modificó las competencias relativas a la administración de los recursos. A partir de esta ley, el antiguo Ministerio de la Protección Social ${ }^{12}$ sería el encargado de girar directamente, en nombre de los entes territoriales, los recursos para financiar la unidad de pago por capitación a las entidades promotoras de salud y/o los servicios que hayan proporcionado las instituciones prestadoras de salud.

Lo anterior significaría que ahora los gobiernos subnacionales debían concentrar su capacidad institucional en la identificación, actualización y registro de los afiliados al régimen subsidiado, de tal forma que dicha información le permitiera al Gobierno nacional realizar giros directos. Así las cosas, pese a que los recursos no ingresarían a la tesorería territorial, se preservaría la autonomía regional sobre la administración del régimen subsidiado ${ }^{13}$.

\footnotetext{
- "Por la cual se definen rentas de destinación específica para la salud, se adoptan medidas para promover actividades generadoras de recursos para la salud, para evitar la evasión y la elusión de aportes a la salud, se redireccionan recursos al interior del sistema de salud y se dictan otras disposiciones."

11 "Por medio de la cual se reforma el Sistema General de Seguridad Social en Salud y se dictan otras disposiciones".

12 Que cambió de nombre por el de Ministerio de Salud y Protección Social con la entrada en vigor de la Ley 1444 de 2011.
} 
Vale aclarar que el giro directo no buscó inyectar capital al sistema de salud, sino agilizar el flujo de recursos que hacían parte del mismo, particularmente porque se observaban altos niveles de endeudamiento, originados principalmente en contratos de administración del régimen subsidiado que habían sido suscritos entre los entes territoriales y las entidades promotoras de salud. Situación que generó eventos de iliquidez, círculos viciosos interinstitucionales y finalmente afectó el acceso de los afiliados a los servicios de salud.

Sumado a lo anterior y ante las condiciones de insostenibilidad financiera que presentaban gran parte de los hospitales públicos conformados como empresas sociales del Estado, el Gobierno nacional, a través de la misma Ley 1438 de 2011, estableció los criterios y las condiciones para la adopción de programas de saneamiento fiscal y financiero bajo el acompañamiento de las direcciones departamentales o distritales de salud.

Así, determinó que todas aquellas empresas Sociales del Estado que, de acuerdo con la evaluación del Ministerio de Salud y Protección Social, presentaran débiles condiciones de mercado, equilibrio, viabilidad financiera y bajos indicadores de salud, y por tanto, fueran categorizadas en riesgo medio o alto, deberían adoptar un programa de saneamiento fiscal y financiero ${ }^{14}$.

Según la norma, el incumplimiento del Programa podría generar acciones de mediación por parte del Gobierno nacional que iban desde la suscripción de un acuerdo de reestructuración de pasivos, o la intervención por parte de la Superintendencia Nacional de Salud, hasta la liquidación o fusión de la entidad.

Para el año 2013, de 967 empresas sociales del Estado evaluadas por el Ministerio de Salud y Protección Social, 540 (56\%) fueron categorizadas en riesgo ${ }^{15}$, de las cuales $412(76 \%)$ presentaban riesgo alto y 128 (24\%) medio. En 2014 fueron categorizados en riesgo 42 hospitales más ${ }^{16}$, lo que refleja un incremento de $10 \%$ respecto al reporte del año anterior. De los

13 Para este caso particular, vale precisar las dimensiones de la descentralización, pues las entidades territoriales conservaron la autonomía administrativa para el seguimiento y control del régimen subsidiado, pero se les redujo el grado de libertad sobre la autonomía financiera.

14 Posteriormente el artículo $8^{\circ}$ de la Ley 1608 de 2013 definió los Programas de Saneamiento Fiscal y Financiero como "un programa integral, institucional, financiero y administrativo que cubre la Empresa Social del Estado, que tiene por objeto restablecer su solidez económica y financiera de estas Empresas, con el propósito de asegurar la continuidad en la prestación del servicio público de salud.

El Programa de Saneamiento Fiscal y Financiero deberá contener medidas de reorganización administrativa, racionalización del gasto, restructuración de la deuda, saneamiento de pasivos y fortalecimiento de los ingresos de las Empresas Sociales del Estado; que permitan su adecuada operación, con el fin de garantizar el acceso, oportunidad, continuidad y calidad en la prestación de los servicios de salud a la población usuaria."

15 Según Resolución 1877 de 2013 del Ministerio de Salud y Protección Social, "Por medio de la cual se efectúa la categorización del riesgo de las Empresas Sociales del Estado del nivel territorial para la vigencia 2013. 
últimos, presentaban riesgo alto 31 (74 \%) y medio 11 (26\%). En suma, 454 empresas sociales del Estado debían adoptar un Programa de Saneamiento Fiscal y Financiero en los términos definidos por el Ministerio de Hacienda y Crédito Público ${ }^{17}$.

Para la financiación de los programas de saneamiento de los hospitales públicos, el Gobierno nacional autorizó ${ }^{18}$ el uso de los excedentes de las cuentas maestras por parte de las entidades territoria$l \mathrm{~s}^{19}$, asignando para ello aproximadamente $\$ 379$ mil millones (equivalentes a un $27 \%$ de los excedentes).

Adicionalmente asignó \$645 mil millones (un $46 \%$ de los excedentes) para la financiación de infraestructura y dotación de la red pública hospitalaria, cuyo rediseño y modernización era ya era una necesidad desde la promulgación de la Ley 1438 de 2011.

De igual forma, aunque la Ley 1438 había ordenado la liquidación de los contratos de régimen subsidiado e implementado el giro directo, las entidades territoriales aún contaban con altos niveles de endeudamiento por concepto de aseguramiento, dado que una parte de dicho régimen se financiaba con recursos propios, por lo que el gobierno asignó \$103 mil millones (7\% de los excedentes) para la financiación de los compromisos adquiridos entre las vigencias 2011 y 2013.

Además, fue aprobado el uso de $\$ 263.000$ millones (19\% de los excedentes) para costear los servicios prestados a la población pobre no asegurada y para el pago de los servicios no incluidos en el plan de beneficios del régimen subsidiado.

Asimismo, se le permitió al Ministerio de Salud y Protección Social asignar a los municipios de categorías 4, 5 y 6 los recursos del FOSYGA hasta por un monto equivalente a $\$ 150.000$ millones para atender las deudas reconocidas del régimen subsidiado ${ }^{20}$. Para el final del año 2014, dicho Ministerio había realizado giros directos por cerca de $\$ 112.000$ millones $^{21}$.

\footnotetext{
16 Según Resolución 2090 de 2014 del Ministerio de Salud y Protección Social, "Por medio de la cual se efectúa la categorización del riesgo de las Empresas Sociales del Estado del nivel territorial para la vigencia 2014 y se dictan otras disposiciones.

17 Mediante el Decreto 1141 de 2013 el Gobierno Nacional estableció los parámetros generales de viabilidad, monitoreo, seguimiento y evaluación de los Programas de Saneamiento Fiscal y Financiero que deben adoptar las Empresas Sociales del Estado del nivel territorial, categorizadas en riesgo medio o alto.

18 Artículo 2 de la Ley 1608 de 2013.

19 Al término del año 2012 los municipios contaban con excedentes en las cuentas maestras del régimen subsidiado que ascendían a 1,4 billones, según el informe del Ministerio de Salud y Protección Social sobre el plan de aplicación y ejecución de los excedentes de las cuentas maestras del régimen subsidiado.

20 Según la Ley 1608 del año 2013, los municipios beneficiarios deberán restituir los recursos en no más de 10 años mediante el incremento del esfuerzo propio en la cofinanciación del régimen subsidiado.

21 Según Resoluciones de giro de recursos operación FOSYGA 2013 y 2014 del Ministerio de Salud y Protección Social.
} 
Como medida para solucionar temporalmente los problemas de liquidez que sufría el sistema de salud, se autorizó al Ministerio de Salud la compra de cartera reconocida por instituciones prestadoras de servicios de salud con empresas promotoras de salud tanto del régimen contributivo como del subsidiado, operaciones que serían financiadas con los recursos de la subcuenta de garantías del FOSYGA. Según cifras del Ministerio, al cierre de la vigencia 2014 se habían realizado 900 operaciones de compra de cartera por un poco más de $\$ 1$ billón, de esta suma aproximadamente el $46 \%$ fue realizado con instituciones prestadoras de servicios de salud públicas o de capital mixto.

Finalmente, considerando que el sistema de salud no solo requería la capitalización de nuevas fuentes de financiación, sino mejorar los flujos de recursos que hacían parte del sistema, el Gobierno nacional creó el Fondo para el Salvamento y Garantías para el Sector Salud (Fonsaet) ${ }^{22}$, el cual financiaría las obligaciones que no hubiesen podido ser canceladas por las empresas sociales del Estado intervenidas o que se encontraran categorizadas en riesgo medio o alto. Además, le permitió al fondo la compra y comercialización de la cartera de las instituciones prestadoras de servicios de salud públicas.

Con todo lo anterior, las cifras del sector parecen no evidenciar la magnitud del problema, pues si bien las medidas del Gobierno nacional se han concentrado principalmente en lo financiero, el balance de los fondos departamentales de salud mostró un superávit de aproximadamente $\$ 1,08$ billones en la vigencia 2013 (Dirección de Apoyo Fiscal, 2015a).

Las expectativas están puestas en la reforma estatutaria presentada por el Gobierno nacional, la cual promete garantizar la salud como derecho fundamental y plantea un nuevo esquema o modelo que se fundamenta esencialmente en la creación de dos entidades: Salud-Mía, entidad pública que tendrá autonomía administrativa, presupuestal y patrimonio independiente, y que administrará los recursos del sistema de la salud; y los gestores de servicios de salud, entidades públicas y privadas que deberán estar adscritas a un área de gestión sanitaria y conformar redes para la prestación de servicios de salud (Sarmiento et al., 2005)

Adicionalmente, según la reforma, se eliminará el Plan Obligatorio de Salud, para darle paso a un nuevo plan de beneficios integral denominado Mi-Plan, el cual deberá incluir todas las patologías de manera que no sea necesario volver a los antiguos comités técnico-científicos. No obstante, definió un listado de exclusiones de servicios, procedimientos y medicamentos, cuya función sea exclusivamente cosmética

22 Fue creado mediante la Ley 1438 del año 2011 y posteriormente modificado por la Ley 1608 del año 2013. 
o suntuaria, así como tecnologías en salud que no cuenten con evidencia científica sobre su seguridad y eficacia.

Así las cosas, Salud-Mía verificará que los Gestores estén garantizando la prestación de los servicios a la población y realizará pagos directos a las instituciones prestadoras de servicios de salud con base en los datos de afiliación que administrará. En ese sentido, hay que precisar que la reforma prevé la transformación de las entidades promotoras de salud, pues aquellas que estén al día con sus obligaciones podrán ser gestoras, en conjunto con las entidades territoriales.

\section{DESPUÉS DE LAS MEDIDAS FI-} NANCIERAS ¿CUÁL FUE EL BALANCE DE LOS FONDOS DEPARTAMENTALES DE SALUD EN 2014?

A continuación se presenta una aproximación global de los resultados presupuestales de los fondos departamentales de salud al término del año 2014, considerando para ello un análisis de fuentes y usos.

\section{Fuentes}

En 2014 los fondos departamentales de salud percibieron ingresos por
$\$ 4,36$ billones, equivalentes al $0,6 \%$ del PIB. La composición por fuentes del sector mostraba en ese año que el $22 \%$ correspondía a rentas tributarias que por disposición legal debían destinarse a la financiación del sector ${ }^{23}$; por otra parte, el $39 \%$ se originó en transferencias, particularmente del nivel central mediante el denominado Sistema General de Participaciones; y el $31 \%$ a ingresos extraordinarios, en su mayor parte compuestos por recursos del balance ${ }^{24}$ (ver Figura 2).

En general las fuentes de financiación del sector salud en los departamentos se concentraron en transferencias e ingresos de capital, hecho que evidencia un nivel importante de dependencia fiscal del nivel central, siendo ello consecuencia de un esquema de descentralización en salud que se fundamenta esencialmente en la distribución de competencias y, de allí, en la definición estricta de reglas de ejecución de los recursos que finalmente han hecho más inflexible el presupuesto de los gobiernos subnacionales.

Los departamentos de Antioquia, Valle del Cauca y Cundinamarca fueron los que mayores recursos percibieron para la financiación del sector, pues agruparon la tercera parte de los ingresos totales; entre tanto, las

\footnotetext{
23 Entre ellas se encuentran rentas nacionales cedidas, como por ejemplo el IVA a los licores, las cervezas y los cigarrillos; así como recursos de esfuerzo fiscal propio de los departamentos que por ordenanza deben invertirse en el sector salud.

24 Agrupa los superávits de vigencias anteriores, la cancelación de reservas presupuestales y los recursos que financian reservas presupuestales constituidas al cierre de la vigencia fiscal del año 2013.
} 


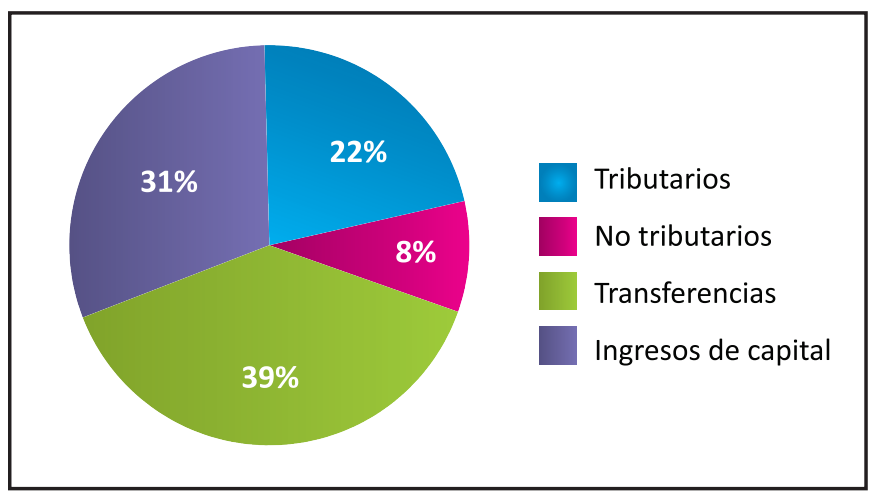

Figura 2. Distribución de fuentes fondos departamentales de salud vigencia 2014 Fuente: elaboración propia con información del Consolidador de Hacienda e Información Pública (CHIP).

entidades de menor presupuesto fueron San Andrés y los antiguos departamentos ${ }^{25}$, cuyos ingresos fueron principalmente transferencias.

Al considerar los ingresos tributarios del sector, los impuestos selectivos al consumo fueron los recursos más importantes para los departamentos, en concreto los impuestos sobre bebidas alcohólicas, cigarrillos y tabaco agruparon el $44 \%$ de los ingresos tributarios; mientras que el IVA sobre bebidas alcohólicas y las estampillas contribuyeron con el $34 \%$ y el $10 \%$, respectivamente (ver Tabla 1).

Tabla 1. Ingresos tributarios departamentales con destino al sector salud (cifras en millones de pesos)

\begin{tabular}{|l|c|c|}
\hline \multicolumn{1}{|c|}{ Concepto } & $\mathbf{2 0 1 4}$ & \% \\
\hline Impuesto a ganadores de sorteos ordinarios y extraordinarios & 18.585 & $2 \%$ \\
\hline Impuesto de loterías foráneas & 16.964 & $2 \%$ \\
\hline Impuesto al consumo de licores, vinos, aperitivos y similares & 64.372 & $7 \%$ \\
\hline Desagregación IVA licores, vinos aperitivos y similares & 321.891 & $34 \%$ \\
\hline Impuesto al consumo cerveza & 259.804 & $27 \%$ \\
\hline Impuesto al consumo de cigarrillos y tabaco & 97.487 & $10 \%$ \\
\hline Impuesto sobre apuestas mutuas & 118 & $0 \%$ \\
\hline Estampillas & 91.555 & $10 \%$ \\
\hline Otros ingresos tributarios & 83.153 & $9 \%$ \\
\hline Total & 953.929 & $100 \%$ \\
\hline
\end{tabular}

Fuente: elaboración propia con información del CHIP.

25 Es decir, Vaupés, Vichada, Guainía, Amazonas y Guaviare. 
De las transferencias al sector, los recursos recibidos del Sistema General de Participaciones representaron el 83 $\%$ de las transferencias totales; entre tanto, los recursos del FOSYGA y del antiguo régimen de regalías ${ }^{26}$ tuvieron una pequeña participación, con el 0,4 $\%$ y el $1 \%$, respectivamente.

Los ingresos de capital constituyeron una porción importante de las fuentes del sector, situación que no es afortunada si se considera que aproximadamente $80 \%$ correspondían a superávits de vigencias anteriores, lo que se traduce en disponibilidades de recursos cercanos a \$1 billón que no fueron invertidos en el sector en la vigencia fiscal en la cual se asignó el presupuesto.

\section{Usos}

En 2014 los departamentos invirtieron $\$ 3,48$ billones en el sector salud, equivalente al 0,5\% del PIB. De dicha suma, el $22 \%$ se ejecutó en el aseguramiento al régimen subsidiado, $12 \%$ en acciones de salud pública, $38 \%$ en la prestación de servicios a la población pobre y $28 \%$ en otros gastos en salud.

Del componente del régimen subsidiado, el rubro más importante fue la cobertura de aseguramiento en salud, con el $67 \%$ de la ejecución (\$517.280 millones). En salud pública, las principales cuentas de inversión fueron el tratamiento de enfermedades transmisibles y zoonosis (un $22 \%$ ), seguridad sanitaria y del ambiente (un 16 $\%$ ), y gestión para el desarrollo operativo del Plan Nacional de Salud Pública (el $20 \%)$.

En relación con el componente de la prestación de servicios, los rubros de inversión más representativos fueron los servicios de salud a la población pobre no asegurada $(67 \%)$ y los servicios no incluidos en el POS del régimen subsidiado (17\%).

El gasto per cápita en salud de los departamentos fue de aproximadamente $\$ 87.130$. Las entidades más pequeñas en términos poblacionales contaron con unas inversiones per cápita más altas, como fue el caso de los antiguos departamentos y San Andrés (ver la Figura 3); sin embargo, entidades como Cundinamarca $^{27}$ y Antioquia, que cuentan con los niveles poblacionales más altos del país, también muestran unos gastos per cápita por encima del indicador departamental. Esta situación puede reflejar la concentración en estas áreas geográficas de los servicios de salud de mayor complejidad.

Contrasta la situación de los departamentos de Bolívar y del Atlántico,

\footnotetext{
26 Establecido por la Ley 141 de 1994. Actualmente las regalías son administradas por el nuevo Sistema General de Regalías, según lo dispuesto por la Ley 1530 de 2012 y demás decretos reglamentarios.

27 Sin incluir el Distrito Capital de Bogotá.
} 


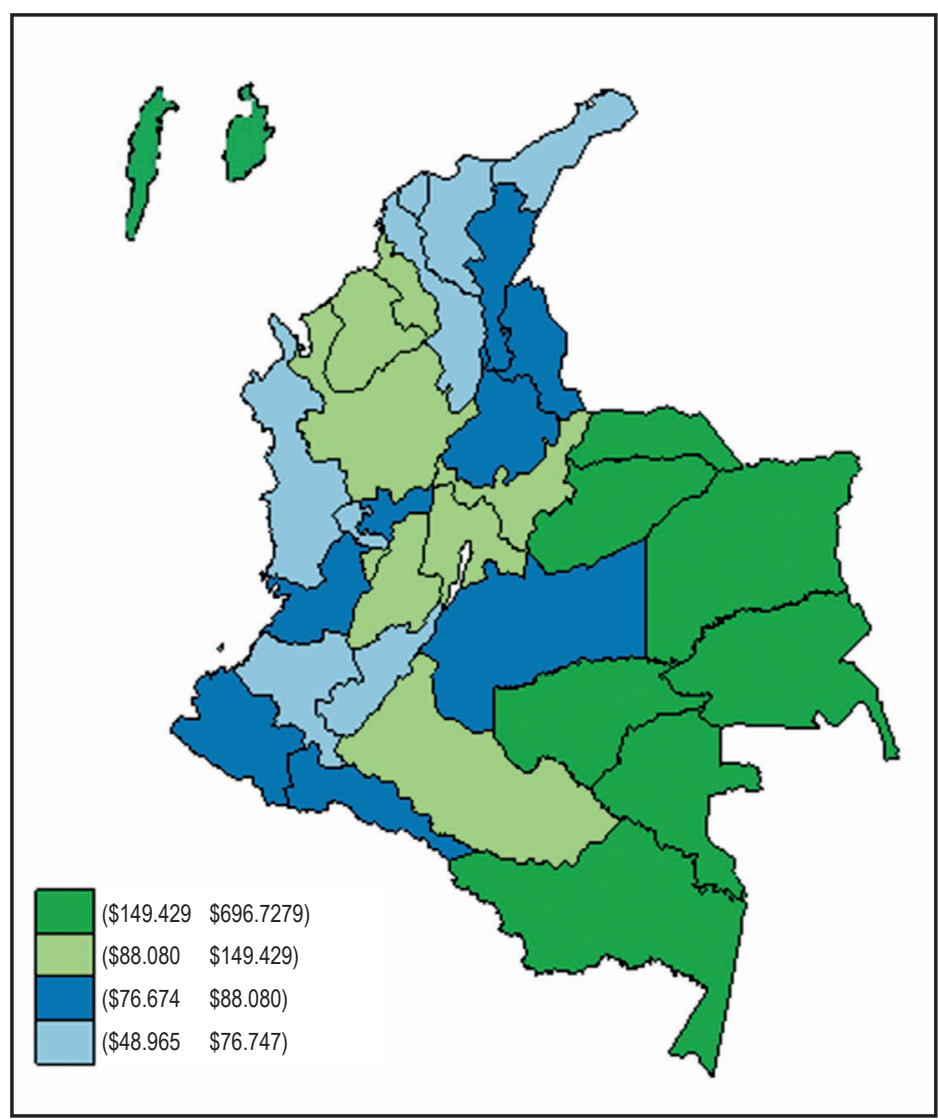

Figura 3. Gasto per cápita fondos departamentales de salud año 2014 Fuente: elaboración propia con información del CHIP y del DANE.

cuyos niveles de inversión per cápita fueron los más bajos del país, pese a que cuentan con tamaños de poblaciones similares a Cundinamarca y Santander.

\section{Balance presupuestal de los departa- mentos}

Al cierre del ejercicio presupuestal del año 2014, los fondos departamentales de salud presentaron un superávit de
$\$ 889.198$ millones (ver Tabla 2), equivalente al $0,1 \%$ del PIB. Pese a que dicho resultado refleja una reducción nominal de $21 \%$ frente al 2013, evidencia que el sector salud en el nivel departamental no ha mostrado problemas financieros estructurales. Por el contrario, se ha caracterizado por mantener disponibilidades de recursos presupuestales de años anteriores, sin la debida ejecución en el periodo fiscal en que se programó la inversión. 
Tabla 2. Resultado consolidado fondos departamentales de salud año 2014 (cifras en millones de pesos)

\begin{tabular}{|l|c|}
\hline \multicolumn{1}{|c|}{ Concepto } & 2014 \\
\hline Total ingresos & 4.364 .388 \\
\hline Total gastos & 3.475 .189 \\
\hline Superávit presupuestal & 889.198 \\
\hline
\end{tabular}

Fuente: elaboración propia con información del CHIP.

De manera general, los departamentos no mostraron tendencia al equilibrio presupuestal, curiosamente no por vía del sesgo al déficit, sino por cuenta de significativos excedentes de recursos que alertan sobre la capacidad de gestión de los entes territoriales para la inversión de los recursos asignados al sector salud.

Lo anterior se reafirma al observar que solo los departamentos de San Andrés y Antioquia exhibieron déficit presupuestal, mientras que Valle del Cauca

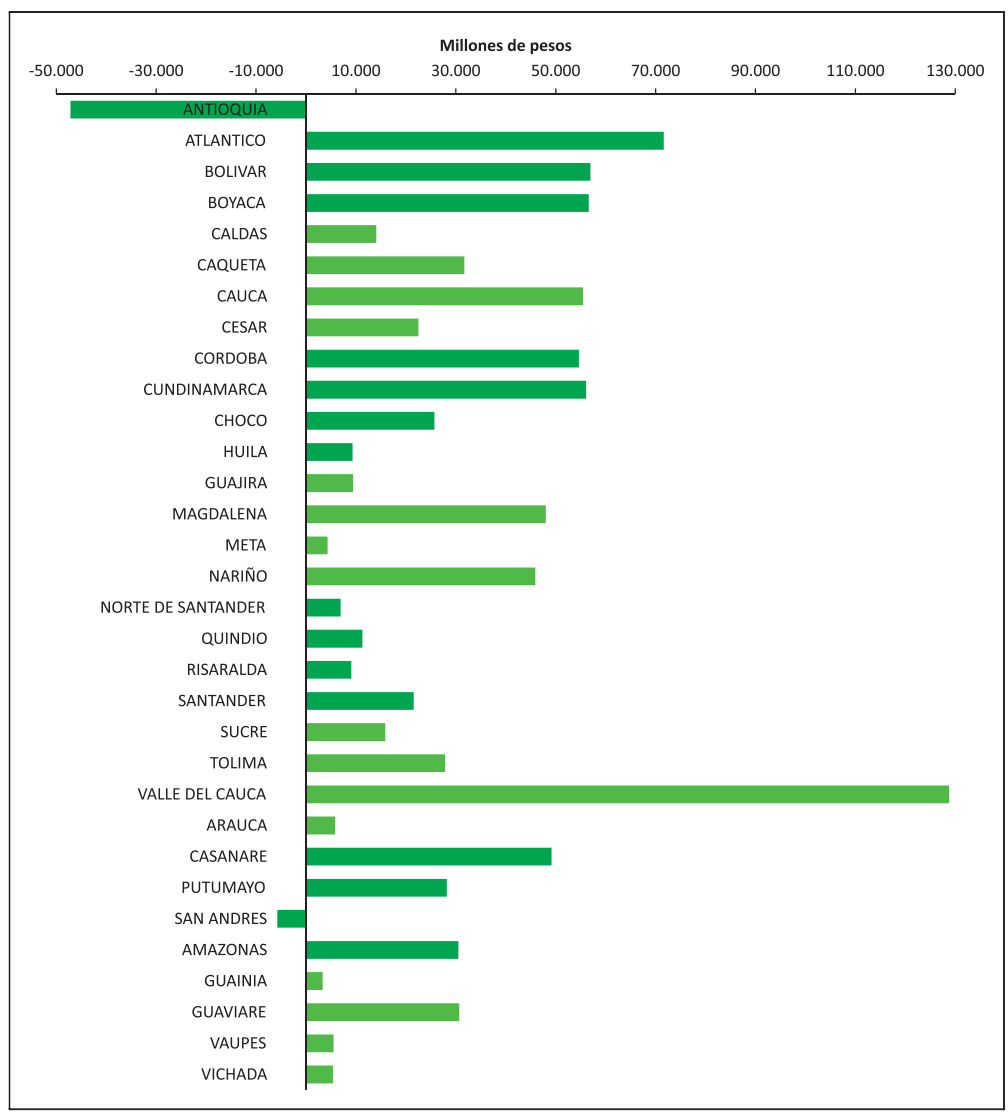

Figura 4. Resultado presupuestal fondos departamentales de salud vigencia 2014 Fuente: elaboración propia con información del CHIP. 
y Atlántico contribuyeron significativamente al superávit consolidado (ver Figura 4). Situación que contrasta con el gasto en salud per cápita, al observar que Atlántico, Magdalena y Chocó presentaron los indicadores más bajos del país, aun cuando sus balances presupuestales fueron positivos.

\section{¿CRISIS FINANCIERA O CRI- SIS DE GESTIÓN?}

Inicialmente los servicios no incluidos en el POS se reclamaban mediante el mecanismo de la tutela, situación que generó altos niveles de déficit fiscal en las entidades territoriales. Con posterioridad a la homologación del POS ordenada por la Corte Constitucional, se conservaba buena parte del déficit, toda vez que existió un rezago ${ }^{28}$ entre la inclusión de los servicios de salud en el plan de beneficios del régimen subsidiado y la igualación de la unidad de pago por capitación (UPC) con el régimen contributivo.

De acuerdo con un informe de la Defensoría del Pueblo (2014), en 2013 se interpusieron 454.500 tutelas, de las cuales el 25,33 \% (115.147) reclamaba servicios de salud, cifra que representó un incremento de $0,73 \%$ frente al año anterior. Adicionalmente, con ocasión de la unificación del POS, el porcentaje de solicitudes mediante tutelas por servicios incluidos en el POS no se redujo y fue más representativo en el régimen subsidiado que el contributivo $(75,63 \%$ y $64,90 \%$, respectivamente), situación que evidencia que aún existían barreas de acceso para algunos servicios, pese a la homologación del POS.

Frente al No POS, hay que precisar que en el régimen subsidiado no se han vuelto a observar porcentajes de tutelas en niveles tan altos como los que se presentaban en el periodo comprendido entre 2006 y $2008^{29}$; sin embargo, con posterioridad se evidenció una tendencia creciente, cerrando el año 2013 con un $24 \%$ de solicitudes que reclamaban servicios no incluidos en el POS.

Lo anterior demuestra que, luego de la homologación de los planes de beneficios, se han venido trasladando solicitudes que se hacían en el No POS subsidiado al POS unificado (Defensoría del Pueblo, 2014). Situación que explica en gran parte el déficit de $\$ 433.781$ millones por prestación de servicios que mostraron los departamentos al cierre de la vigencia 2013 (Dirección General de Apoyo Fiscal, 2015a).

Así las cosas, uno de los desafíos de la reforma a la salud será entonces llevar a la práctica la unificación del plan de beneficios, así como la igualación de los mecanismos de financiación de los

28 La unificación del POS inició efectivamente a partir de julio de 2012.

29 En promedio el 69 \% de las tutelas solicitaban servicios no incluidos en el POS. 
servicios, de manera tal que se elimine la presión que ha venido ejerciendo el tren de gastos No POS sobre las finanzas públicas subnacionales.

Como se observa en la Figura 2, Colombia ha avanzado considerablemente en el objetivo de la cobertura universal en salud; sin embargo, ello se ha logrado con una porción significativa en afiliación al régimen subsidiado $^{30}$, situación que debe ser permanentemente evaluada toda vez que el modelo de financiación del sistema de salud promovido desde la Ley 100 de 1993 ha requerido que las entidades territoriales avancen hacia una senda sostenible de crecimiento económico, con bondades sobre la generación de empleo y la formalización laboral, de manera que los mecanismos de financiación a través de las contribuciones salariales, rentas cedidas y las transferencias del Gobierno nacional permitan mantener el esquema de subsidios cruzados (Salazar, 2011).

Pese a que el sistema de salud ha recibido significativas inyecciones de recursos, el sector no vislumbrará mejoras sustanciales en términos de sostenibilidad financiera a medio y largo plazo, si no se materializa un proceso de fortalecimiento de la capacidad institucional de las administraciones territoriales que permita optimizar la asignación y flujo interinstitucional de recursos, garantizar calidad en la ejecución de la inversión, reorganizar y articular las redes de prestación de servicios desde una perspectiva regional y con facilidades de acceso a la población, y mejorar los sistemas de información locales de forma que facilite la coordinación de la política pública entre el Gobierno nacional y subnacional y haga más efectivos los instrumentos de seguimiento y control sobre la prestación de los servicios de salud.

Por otra parte, la efectividad de los programas dirigidos a lograr el saneamiento fiscal y financiero de las empresas sociales del Estado no solo se medirá con el saneamiento de pasivos del sector, sino con la continuidad en el tiempo de las reglas fiscales cuantitativas que se hayan establecido de acuerdo con las condiciones propias de cada hospital, de forma que sea factible proyectar un marco financiero sostenible en la redes públicas de prestación de servicios de salud.

Los cuantiosos recursos que han sido capitalizados para el sector y el superávit que registraron los fondos departamentales de salud en los últimos años,

\footnotetext{
30 De acuerdo con las estadísticas de afiliación del Ministerio de Salud y Protección Social, a diciembre de 2014 se encontraban afiliados 45.492 .407 personas, de las cuales el $50 \%$ pertenecía al régimen subsidiado, $46 \%$ al contributivo y $4 \%$ al de excepción.
} 

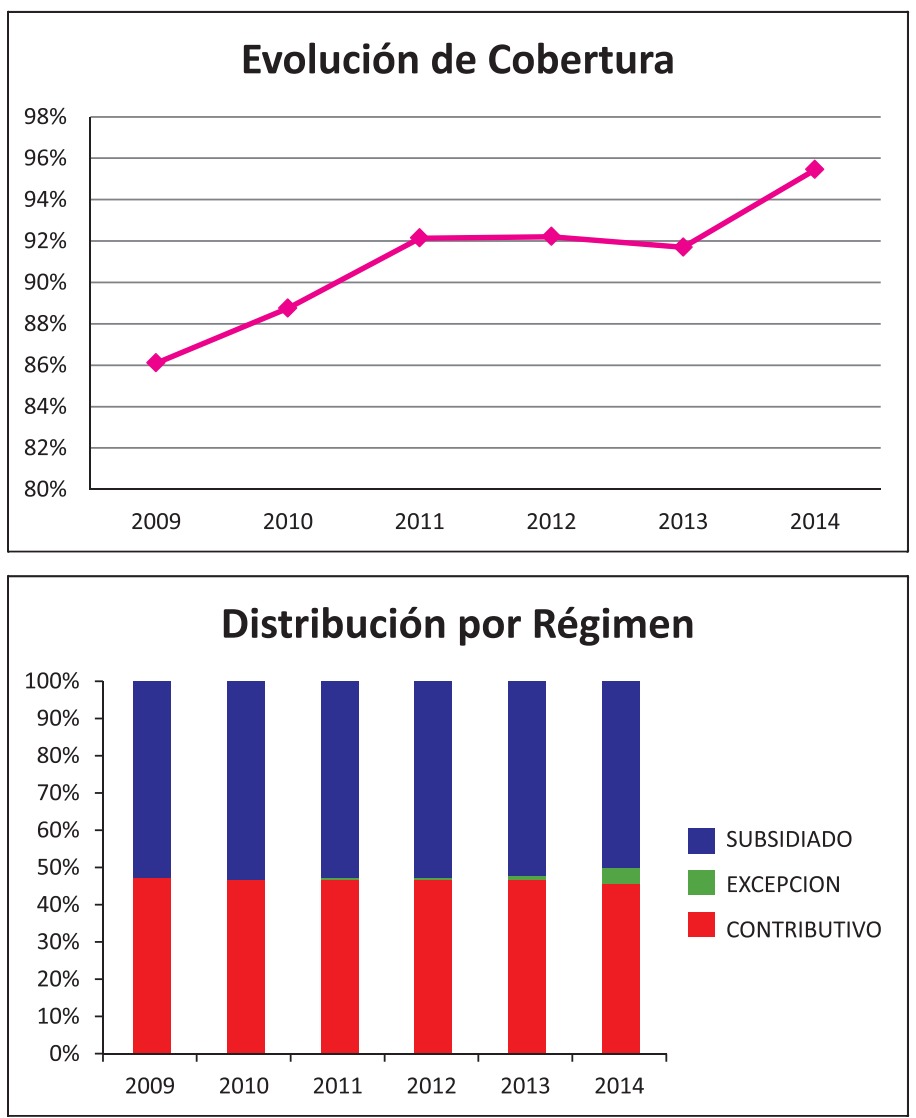

Figura 5. Evolución y distribución del aseguramiento Fuente: elaboración propia con información del Ministerio de Salud y Protección Social y del DANE.

sugieren que ya no se trata de una crisis financiera, sino de una crisis de gestión y de confianza en la institucionalidad del sistema, que ha generado problemas de eficiencia con implicaciones sobre la prestación de los servicios de salud. En ese sentido, la crisis del sector no se resuelve solamente desde una óptica financiera, sino con medidas que fortalezcan la capacidad de gestión de cada uno de los actores del sistema, entre ellos las entidades territoriales.

\section{CONCLUSIONES}

La revisión aquí realizada de la evolución del sector muestra que, hoy en día, el problema financiero perdió importancia. El sistema de salud en 
Colombia presenta características antagónicas: por un lado, como señalan Nuñez et al., (2012), Barón (2007) y Orozco (2015), el sistema de salud colombiano es uno de los esquemas que más recursos tiene en sus arcas para su financiación. Sin embargo, a su vez tiene importantes debilidades y limitaciones en su estructura financiera, que han afectado de forma negativa la prestación del servicio de salud. Ejemplo de ello son los resultados presupuestales superavitarios de los fondos departamentales de salud en 2013, frente al déficit en el componente de prestación de servicios en el mismo año.

Por otra parte, el resultado más destacado políticamente es el aseguramiento en salud, actualmente muy cercano a la cobertura universal (95\%), sin embargo dicho indicador se ha sustentado en una tasa significativa de afiliación al régimen subsidiado, pues para el 2014 aproximadamente el $50 \%$ de la población asegurada pertenecía a dicho régimen. Este hecho hace más vulnerable la sostenibilidad financiera del sistema, dado un contexto económico de bajas perspectivas de crecimiento económi$\mathrm{co}^{31} \mathrm{y}$, por tanto, de riesgos sobre consolidación de un mercado laboral formal que permita fortalecer el régimen contributivo y, con ello, mantener los esquemas de subsidios cruzados en salud.
Finalmente, Colombia aún está en un proceso de consolidación de la descentralización, por lo que la reforma estatutaria de la salud tiene entonces importantes desafíos, particularmente en lo relacionado con el esquema de centralización administrativo y financiero que delinea y, por tanto, las restricciones que ello origina frente a la autonomía y las competencias que tienen las entidades territoriales en materia de salud (Fedesarrollo, 2013).

En todo caso, el sistema de salud requiere de reformas estructurales. No obstante, a diferencia del discurso hasta ahora común, las reformas no deberían estar solamente enmarcadas en el plano financiero, sino especialmente en el rediseño de su estructura institucional. El objetivo debería ser el fortalecimiento de la capacidad de gestión de cada uno de los actores del sistema, a la vez que se facilite la coordinación de la política pública nacional y regional de cara a garantizar que los recursos del sector se administren adecuadamente y que toda la población pueda acceder, sin distinción alguna, a los servicios de salud.

Un punto que puede ser relevante en este contexto es el de políticas como la atención primaria en salud (APS) que podrían proporcionar modelos de gestión adecuados a nivel municipal. Esto, junto con el tratamiento de aquellas variables económicas que afectan

31 En particular por el impacto que ha tenido la dinámica de las regalías sobre los presupuestos territoriales. 
la salud de la comunidad, así como los llamados determinantes sociales de la enfermedad, (De Santis \& Villagra, 2014) pueden ser más fácilmente abordables a nivel de los entes territoriales debido al tamaño de la población. Alternativas de este tipo podrían contribuir a una mejor gestión en este tipo de entes.

Vale la pena resaltar que los elementos aquí encontrados se refieren a la salud, pero coinciden con lo encontrado por otros autores en materia fiscal territorial. Chamorro y Urrea (2016), en un estudio sobre la incidencia de las reglas fiscales a nivel territorial, encontraron un hecho estilizado: que la tendencia deficitaria en los entes territoriales se ha revertido. Si durante la década del 90 los gastos crecieron más rápido que los ingresos, a partir de 2000 las finanzas de los entes territoriales se recuperaron y se vincularon más al ciclo político. Es posible que lo ocurrido en el sector salud ayude a explicar este fenómeno.

Aunque esta situación se puede explicar por la aplicación del marco de responsabilidad fiscal, lo cierto es que el sector salud ha venido acumulando una cuantía importante de recursos sin ejecutar. El informe de la Dirección de Apoyo Fiscal del Ministerio de Hacienda (2015b) muestra que los departamentos reportaron en el 2014 un saldo acumulado de disponibilidades netas del sistema general de participaciones $^{32}$ para el sector salud por alrededor de 557.000 millones de pesos. Aunque se trata de recursos con destinación específica establecida por la Ley 715, estos montos contribuyen a cumplir en el agregado con las metas de responsabilidad fiscal.

De cara a la reforma del sistema general de participaciones se discute sobre la conveniencia de mantener el esquema inflexible de destinaciones específicas o la necesidad de hacer distribuciones más generales que le permitan a la entidad territorial tener discrecionalidad sobre la ejecución de los recursos de salud teniendo en cuenta sus necesidades.

En todo caso, los aspectos relativos a la situación fiscal territorial y la gestión de la salud merecen seguir siendo estudiados en detalle.

32 Las disponibilidades netas corresponden a la diferencia entre los activos de fácil realización y las exigibilidades y reservas presupuestales. 


\section{REFERENCIAS}

Almeida, C. (2002). Reforma de sistemas de servicios de salud y equidad en América Latina y el Caribe: Algunas lecciones de los años 80 y 90. Cadernos de Saude Publica, 18(4), 905-925.

Barón, G. (2007). Cuentas de salud de Colombia 1993-2003. Programa de Apoyo a la Reforma de Salud-PARS-. Bogotá: Ministerio de la Protección Social.

Bertranou, F.M. (1999). Are market-oriented health insurance reforms possible in Latin America? The cases of Argentina, Chile and Colombia. Health Policy, 47, 19-36.

Bonet, J., Pérez, G. J. \& Ayala, J. (2014). Contexto histórico y evolución del SGP en Colombia, Documentos de Trabajo Sobre Economía Regional, núm. 205. Cartagena: Banco de la República.

Bottia, M., Cardona-Sosa, L. \& Medina, C. (2012, jul-dic) El SISBEN como mecanismo de focalización individual del régimen subsidiado en salud en Colombia: ventajas y limitaciones. Revista de Economía del Rosario, 15(2), 137-177.

Castellanos, P. (2008). La reforma del sistema público de salud de República Dominica$n a$. The Center for Migration and Development. Working Paper No. 08-051. NJ: Princeton University. Recuperado de https://www.princeton.edu/cmd/working-papers/idlac08/wp08051.pdf

Chamorro, R. A. \& Urrea, A. F. (2016) Incidencia de las reglas fiscales en la sostenibilidad de la deuda pública territorial en Colombia. Cuadernos de Economía, 35(67), 207-251.

Congreso de la República de Colombia. (1993, 23 de dic.). Ley 100 de 1993, por la cual se crea el sistema de seguridad social integral y se dictan otras disposiciones. Bogotá: Imprenta Nacional.

Congreso de la República de Colombia. (2001, 21 de dic.). Ley 715 de 2001, por la cual se dictan normas orgánicas en materia de recursos y competencias de conformidad con los artículos 151, 288, 356 y 357 (Acto Legislativo 01 de 2001) de la Constitución Política y se dictan otras disposiciones para organizar la prestación de los servicios de educación y salud, entre otros. Bogotá: Imprenta Nacional.

Congreso de la República de Colombia. (2007, 9 de ene.). Ley 1122 de 2007, por la cual se hacen algunas modificaciones en el Sistema General de Seguridad Social en Salud y se dictan otras disposiciones. Bogotá: Imprenta Nacional.

Congreso de la República de Colombia. (2007, 27 de dic.). Ley 1176 de 2007, por la cual se desarrollan los artículos 356 y 357 de la Constitución Política y se dictan otras disposiciones. Bogotá: Imprenta Nacional. 
Congreso de la República de Colombia. (2010, 12 de jul.). Ley 1393 de 2010, por la cual se definen rentas de destinación especifica para la salud, se adoptan medidas para promover actividades generadoras de recursos para la salud, para evitar la evasión y la elusión de aportes a la salud, se redireccionan recursos al interior del sistema de salud y se dictan otras disposiciones. Bogotá: Imprenta Nacional.

Congreso de la República de Colombia. (2011, 19 de ene.). Ley 1438 de 2011, por medio de la cual se reforma el Sistema General de Seguridad Social en Salud y se dictan otras disposiciones. Bogotá: Imprenta Nacional.

Congreso de la República de Colombia. (2011, 4 de mayo). Ley 1444 de 2011, por medio de la cual se escinden unos Ministerios, se otorgan precisas facultades extraordinarias al Presidente de la República para modificar la estructura de la Administración Pública y la planta de personal de la Fiscalía General de la Nación y se dictan otras disposiciones. Bogotá: Imprenta Nacional.

Congreso de la República de Colombia. (2013, 2 de ene.). Ley 1608 de 2013, por medio de la cual se adoptan medidas para mejorar la liquidez y el uso de algunos recursos del Sector Salud.Bogotá: Imprenta Nacional.

De Santis, M. \& Villagra, I. (2014) Condiciones económicas y capital social como determinantes de la salud y el bienestar subjetivo. El caso de Argentina durante 1995 y 1996. Cuadernos de Economía, 33(63), 543-567.

Defensoría del Pueblo Colombia. (2014). La tutela y los derechos a la salud y a la seguridad social 2013. Bogotá: Imprenta Nacional de Colombia.

Dirección General de Apoyo Fiscal, Ministerio de Hacienda y Crédito Público. (2014). Responsabilidad fiscal subnacional y descentralización en Colombia: quince años de consolidación. Bogotá D.C. Recuperado de http://www.minhacienda.gov.co/ portal/page/portal/HomeMinhacienda/asistenciaentidadesterritoriales/Publicaciones/Financieras/15\%20anios.pdf

Dirección General de Apoyo Fiscal, Ministerio de Hacienda y Crédito Público. (2015a). El sector salud en las entidades territoriales y propuestas de ajuste a la ley de transferencias. Bogotá D.C. Recuperado de http://www.minhacienda.gov.co/HomeMinhacienda/asistenciaentidadesterritoriales/Publicaciones/recursosSGP/CartillaSaludFinal.pdf

Dirección General de Apoyo Fiscal, Ministerio de Hacienda y Crédito Público. (2015b). Informe sobre la viabilidad fiscal de los departamentos. Vigencia 2014. Recuperado de http://www.minhacienda.gov.co/portal/page/portal/HomeMinhacienda/asistenciaentidadesterritoriales/Publicaciones/Financieras/IVF_AAA_INTRODUCCION_2014.pdf

Fedesarrollo. (2013). La Ley Estatutaria de Salud: ¿legislando para arcángeles sin restricción de presupuesto? Tendencia Económica, 130. 
Frenk, J., González-Pier, E., Gómez-Dantés, O., Lezana, M. \& Knaul, F. M. (2006). Comprehensive Reform to improve Health System Performance in Mexico. The Lancet, 368, 1524-1534.

Knaul, F. M., Gonzalez-Pier, E., Gómez Dantés, O., García-Junco, D., Arreola-Ornelas, H., et al. (2012) The Quest for Universal Health Coverage: Achieving Social Protection for All in Mexico. Health Policy, 380, 1-21.

Londoño, J. L. \& Frenk, J. (1997) Structured Pluralism: Towards an Innovative Model for Health System Reform in Latin America. Health Policy, 41(1), 1-36.

Melo, L. A. \& Ramos, J. E. (2010). Algunos aspectos fiscales y financieros del sistema de salud en Colombia. Borradores de economía, Banco de la República.

Ministerio de Hacienda y Crédito Público. (2009). 10 años de transformación fiscal territorial en Colombia. Bogotá: Imprenta Nacional de Colombia. Recuperado de http:/www.minhacienda.gov.co/portal/page/portal/HomeMinhacienda/asistenciaentidadesterritoriales/Publicaciones/Financieras/10\%20A\%D1OS\%20 TRANSFORMACION\%2010-06.pdf

Ministerio de Hacienda y Crédito Público. (2011). Monitoreo, seguimiento y control al uso de los recursos del sistema general de participaciones vigencia 2011. Bogotá: Imprenta Nacional de Colombia. Recuperado de http://www.minhacienda.gov.co/HomeMinhacienda/asistenciaentidadesterritoriales/Publicaciones/recursosSGP/MONITOREO_Y_SEGUIMIENTO.pdf

Ministerio de Salud y Protección Social, República de Colombia (2013, 30 de mayo). Resolución 1877 de 2013, por medio de la cual se efectúa la categorización del riesgo de las Empresas Sociales del Estado del nivel territorial para la vigencia 2013. Bogotá: Minsalud.

Ministerio de Salud y Protección Social, República de Colombia (2014, 29 de mayo). Resolución 2090 de 2014, por medio de la cual se efectúa la categorización del riesgo de las Empresas Sociales del Estado del nivel territorial para la vigencia 2014 y se dictan otras disposiciones. Bogotá: Minsalud.

Núñez, J., Zapata, J. G., Castañeda, C., Fonseca, M. \& Ramírez, J. (2012). La sostenibilidad financiera del sistema de salud colombiano - dinámica del gasto y principales retos de cara al futuro. Bogotá: Fedesarrollo.

Nupia, O. A. \& Sánchez, F. (2001). Eficiencia en los hospitales públicos de Bogotá. Desarrollo y Sociedad, Universidad de los Andes, 101-136.

Orozco-Gallo A. J. (2015) Un análisis del gasto público en salud de los entes territoriales Colombianos. Documentos de Trabajo Sobre Economía Regional. Cartagena, Colombia: Banco de la República, Centro de Estudios Económicos Regionales de Cartagena. 
Presidente de la República de Colombia (2008, 10 de ene.). Decreto 028 de 2008, por medio del cual se define la estrategia de monitoreo, seguimiento y control integral al gasto que se realice con recursos del Sistema General de Participaciones. Bogotá: Imprenta Nacional.

Presidente de la República de Colombia (2013, 31 de mayo). Decreto 1141 de 2013, por el cual se determinan los parámetros generales de viabilidad, monitoreo, seguimiento y evaluación de los Programas de Saneamiento Fiscal y Financiero que deben adoptar las Empresas Sociales del Estado del nivel territorial, categorizadas en riesgo medio o alto y se dictan otras disposiciones. Bogotá: Imprenta Nacional.

Salazar, N. (2011). El régimen de seguridad social en salud: problemas financieros, medidas adoptadas y retos para el futuro próximo. Notas Fiscales, No. 6. Bogotá: Ministerio de Hacienda y Crédito Público.

Sarmiento-Gómez, A., Castellanos-Aranguren, W., Nieto-Rocha, A. C., Alfonso-Malaver, C. E. \& Pérez-García, C. A. (2005). Análisis de eficiencia técnica de la red pública de prestadores de servicios dentro del Sistema General de Seguridad Social en Salud. Archivos de Economía. Bogotá: Departamento Nacional de Planeación.

Stiglitz, J. E. (2000). La economía del sector público (3 ed.). M. Rabasco, \& L. Toharia (trads.) Barcelona, España: Antoni Bosch.

Urrutia, M. \& Llano, J. (2012). Los actores en la crisis económica de fin de siglo. Bogotá: Uniandes. 